Article

\title{
Evaluation of Loofah Lines for Resistance to Tomato Leaf Curl New Delhi Virus and Downy Mildew, as well as Key Horticultural Traits
}

\author{
Narinder P. S. Dhillon ${ }^{1, *}$, Mohammed Abu Taher Masud ${ }^{2}$, Somchit Pruangwitayakun ${ }^{1}$, \\ Monpapa Natheung ${ }^{1}$, Suyuporn Lertlam ${ }^{1}$ and Robert L. Jarret ${ }^{3}$ \\ 1 World Vegetable Center, East and Southeast Asia, Kasetsart University, Kamphaeng Saen, \\ Nakhon Pathom 73140, Thailand; somchit.pruangwitayakun@worldveg.org (S.P.); \\ osaki_puk@hotmail.com (M.N.); taily5919@gmail.com (S.L.) \\ 2 Vegetable Division, Horticultural Research Center, Bangladesh Agricultural Research Institute, Joydebpur, \\ Gazipur 1701, Bangladesh; abt_masud@yahoo.com \\ 3 U.S. Department of Agriculture, Agricultural Research Service, Plant Genetic Resources Conservation Unit, \\ 1109 Experiment St., Griffin, GA 30223, USA; bob.jarret@usda.gov \\ * Correspondence: narinder.dhillon@worldveg.org
}

Received: 19 May 2020; Accepted: 13 July 2020; Published: 15 July 2020

\begin{abstract}
Two loofah (Luffa) species, the ridge gourd (Luffa acutangula (L.) Roxb.) and the sponge gourd (L. cylindrica (L.) M. Roem.; syn. L. aegyptiaca), are cultivated widely in Asia by smallholder famers. Both species have significant economic and nutritional importance. However, Tomato leaf curl New Delhi virus (ToLCNDV) and downy mildew (DM) caused by Pseudoperonospora cubensis are important biotic constraints to loofah production throughout Asia. Loofah landrace-derived breeding lines, developed at the World Vegetable Center (WorldVeg), were evaluated at the WorldVeg East and Southeast Asia Research and Training Station, Kasetsart University, Kamphaeng Saen, Thailand-where natural epidemics of ToLCNDV and DM regularly occur. The lines were also evaluated for other commercially important horticultural traits such as days to $50 \%$ staminate and pistillate flowering, fruit color, fruit bitterness, and market segment classification. Thirteen and 59 lines of ridge gourd and sponge gourd, respectively, were determined to be resistant to both ToLCNDV and DM. These lines covered all market segments of loofah and exhibited variability for all of the evaluated horticultural traits. The results of these evaluations and their implications on loofah breeding are discussed.
\end{abstract}

Keywords: Luffa acutangula; Luffa cylindrica; Tomato leaf curl New Delhi virus; downy mildew; resistance; fruit traits; evaluation; germplasm

\section{Introduction}

Ridge gourd (L. acutangula (L.) Roxb.) and sponge gourd (Luffa cylindrica (L.) M. Roem.; syn. L. aegyptiaca Mill.) are the principal cultivated species in the genus Luffa [1]. Seven additional wild species of Luffa include L. graveolens Roxb. (var. longistyla), L. echinata Roxb., L. tuberosa Roxb., L. umbellata Roem, L. quinquefda (Hook and Arn), L. astorii Svans, and L. saccata. In Asia, the two cultivated species (hereafter referred to as loofah) are a commercially and nutritionally significant cucurbitaceous market vegetable. Immature loofah fruit have a significant role in Asian cuisines and are eaten boiled, peeled, and fried, and in curries and soups. Loofah fruits contribute calcium (20 mg/100 g Fresh Weight (FW), magnesium (14 mg/100 g FW), potassium (139 mg/100 g FW), and vitamin A (410 IU) to the human diet [2]. A $200 \mathrm{~g}$ serving of loofah fruit provides between $5 \%$ and $16 \%$ of the daily recommended intake, respectively, of the previously noted nutrients. In addition to their 
nutritional importance, the production of these gourds in Asia provides a livelihood for resource-poor farmers. Loofah can be grown in various agro-climates and the fruits are produced during the hot and wet seasons. The immature fruits exhibit good postharvest, transportation, and storage properties. Within Asia, the sponge gourd fruit typically fall within one of two standard commercial market segments based on fruit color (light green and green), while there are three standard commercial market segments of ridge gourd based on fruit length (short, medium, and long). In addition to its edible immature fruit, the fibrous endocarp of mature fruits (scrubbing sponge) is popular with consumers in the USA, Japan, Korea, and other Asian countries.

Loofah gourds rank high in the cucurbit portfolio of private seed companies. In India alone, the estimated total loofah seed market is $490 \mathrm{MT}$ (ridge gourd: $\mathrm{F}_{1}$ hybrid seed $=80$ tons, open-pollinated $(\mathrm{OP})=80$ tons; sponge gourd: $\mathrm{F}_{1}$ hybrid seed $=200$ tons, $\mathrm{OP}=130$ tons) [3]. The current market in Bangladesh for ridge gourd and sponge gourd seed is 16 and 22 MT, respectively [4]. A recent (2019) survey of Asia Pacific Seed Association (APSA) member companies indicated that loofah should be considered a priority crop for improvement by WorldVeg. The survey predicted moderate to strong growth in the loofah seed market in the next 10 years [5]. Currently, loofah breeding research is centered in India and Bangladesh.

Tomato leaf curl New Delhi virus (ToLCNDV) is a whitefly transmitted bipartite begomovirus [6] initially reported in the Solanaceae, where it caused devastating damage to the tomato crop. This virus was first reported in India in 1995 [6], from where it was disseminated to South and Southeast Asian countries. It remained confined to Asia until 2012, when it was observed infecting cucurbits in various Mediterranean countries such as Spain [7], Tunisia [8], Italy [9], Morocco [10], Greece [11], and Algeria [12]. Very recently, this begomovirus has been reported in cucurbits in Portugal and Estonia [13]. ToLCNDV has caused significant damage to various cucurbitaceous crops, including luffa (L. cylindrica (L.) M. Roem.), wax/ash gourd (Benincasa hispida (Thunb.) Cogn.), cucumber (Cucumis sativus L.), watermelon (Citrullus lanatus L.), melon (C. melo L.), bottle gourd (Lagenaria siceraria (Molina.) Standley), and various kinds of pumpkins/squashes (Cucurbita spp.) [14-18]. The symptoms associated with this virus in cucurbits are shortened internodes, distorted (upward and downward) leaf curling, severe mosaicism with chlorotic leaves, and fruit skin roughness [7]. Severe infections lead to large yield losses and to fruits with a greatly decreased market value.

Pseudoperonospora cubensis, the disease organism causing downy mildew (DM), is a member of the Peronosporaceae. It is a seed-transmitted [19] biotroph or obligate parasite that affects plants of all ages [20]. Infecting only the foliage, the subsequent reduction in the photosynthetic activity early in plant development results in stunted plants and yield reduction [20]. The host range of P. cubensis is extensive and includes 50 species in 20 genera within the Cucurbitaceae. Nineteen host species are in the genus Cucumis [21-23]. The disease is found on Luffa in Southeast Asia [20] and India [24]. Symptoms of DM frequently occur on the foliage, where the infection initially appears as small water-soaked lesions on the underside of the leaves. These lesions are angular and are limited by the leaf veins. They eventually turn from chlorotic to necrotic spots. Environmental conditions play a key role in the disease development [25]. The pathogen flourishes in warm humid regions. Differential test studies have detected several races of $P$. cubensis [26-30]. Six pathotypes of $P$. cubensis have been observed on the basis of their compatibility with specific host genera [31,32]. European populations of P. cubensis have been found to be highly variable and may contain multiple pathotypes [33].

The commercial loofah cultivars currently available in Asia are susceptible to both ToLCNDV and DM. Controlling these pathogens is a major challenge for loofah growers. Although loofah genetic resources naturally exhibit considerable variability for fruit color, size, and other horticultural characteristics, the diversity of the loofah germplasm held by seed companies is extremely limited. Furthermore, loofah genetic resources covering different market segments, and resistant to ToLCNDV and DM, have not been identified. ToLCNDV and DM are both endemic at the World Vegetable Center's (WorldVeg) research station at Kamphaeng Saen, Thailand. The endemic disease pressure at this site provides the WorldVeg loofah breeding program the opportunity to select for ToLCNDV and 
DM resistance. Here, we report the results of the field screening of the WorldVeg loofah breeding lines ( $\mathrm{S}_{5}$ generation) for their reaction to ToLCNDV and DM, and their evaluation for key horticultural traits.

\section{Materials and Methods}

A field screening (two growing seasons per year) of the WorldVeg loofah germplasm collection of landraces consisting of 467 accessions of ridge gourd and 783 accessions of sponge gourd, collected from 10 Asian countries against ToLCNDV and DM, began in 2016 at the WorldVeg East and Southeast Asia Research and Training Station, Kasetsart University, Kamphaeng Saen, Thailand. Populations were initially segregated for resistance to ToLCNDV and DM, and for fruit traits such as color, shape, length, bitterness, and duration of fruit harvest. Plants tolerant to ToLCNDV and DM and belonging to different market segments (bitter vs. non-bitter fruit), were selected and hand-pollinated (selfed) to produce progeny, which were used in the subsequent growing/selection cycles (plant to progeny row). Six plants of each ridge gourd (82) and sponge gourd (65) breeding line (Tables 1 and 2), developed through this breeding approach ( $\mathrm{S}_{5}$ generation), and a susceptible check for both ToLCNDV and DM (20 plants each), were transplanted into single $9.6 \mathrm{~m}^{2}$ plots on 25 December 2019. Natural epidemics of ToLCNDV and DM occurred on the loofah during this period.

Plants were rated in the field 60 days after transplanting for ToLCNDV severity using a 0-2 scale: $0=$ no visible symptoms, $1=$ mild symptoms (slight chlorosis of leaves), and $2=$ severe symptoms (severe chlorosis of leaves, upward leaf curling, and stunting of plants). Severity ratings of 0,1 , and 2 corresponded to resistant, moderately resistant, and susceptible, respectively. To confirm the presence of ToLCNDV, diseased leaf tissue from individual plants of the susceptible checks were crushed in a $500 \mu \mathrm{L}$ Tris-Ethylenediaminetetraacetic acid (TE) buffer, and DNA was extracted as described previously [34]. The quality of the DNA was evaluated on a $1 \%$ agarose gel and stored at $-20^{\circ} \mathrm{C}$ until further use. PCR amplifications were performed using a ToLCNDV-specifc primer pair Beg434F + ToLC1524R (developed by Dr. Orawan Chatchawankanphanich, The National Science and Technology Development Agency (NSTDA), Kasetsart University, Thailand). DNA fragments of the expected size, ca. $1.254 \mathrm{bp}$, were amplified. All of the samples of the control plants collected from the loofah experimental plot were positive, as per the PCR analysis.

DM severity was also assessed 60 days after transplanting. The genotypes were categorized into three groups, namely, immune ( $0 \%)$, resistant (1-10\%), moderately resistant (11-30\%), and susceptible $(>30 \%)$, based on the percentage of symptomatic leaf area (angular lesions), as determined using a modified procedure of that described previously [35]. The presence of Pseudoperonospora cubensis was confirmed based on the culture morphology and microscopic examination of sporangiophores and sporangia as described by Waterhouse and Brothers [36].

The following horticultural traits were recorded: (1) days to $50 \%$ pistillate flowering after transplanting, (2) days to 50\% staminate flowering after transplanting, (3) fruit color, (4) market segment of ridge gourd based on fruit length (short (up to $30 \mathrm{~cm}$ ), medium $(31-44 \mathrm{~cm}$ ), and long $(>45 \mathrm{~cm})$ ), and (5) market segment based on fruit length in sponge gourd (short (up to $20 \mathrm{~cm}$ ), medium $(21-30 \mathrm{~cm})$, and long $(>30 \mathrm{~cm}))$. The number of days from transplanting to the first open pistillate and staminate flowers was recorded for each plant. Fruit bitterness was determined using three fresh fruits of each line that were harvested at marketable maturity. These were washed and cut into small (ca. $3 \mathrm{~g}$ ) pieces (after removing the terminal three inches of each fruit) and used for organoleptic assessment by a three-person taste panel. Two categories of bitterness were recorded-non-bitter and bitter. The evaluators rinsed their mouths with water after each sample tasting. 
Table 1. Summary of disease reaction of lines of ridge gourd (Luffa acutangula) to Tomato leaf curl New Delhi virus (ToLCNDV) and downy mildew (DM), days to 50\% pistillate and staminate flowering, and fruit traits of lines field-evaluated in 2020 at the WorldVeg, Thailand.

\begin{tabular}{|c|c|c|c|c|c|c|c|c|}
\hline Entry & Origin & $\begin{array}{c}\text { Days } 50 \% \\
\text { Pistillate } \\
\text { Flowering } \\
\text { (mean } \pm \text { SE) }\end{array}$ & $\begin{array}{c}\text { Days } 50 \% \\
\text { Staminate } \\
\text { Flowering } \\
\left(\text { mean } \pm \mathrm{SE}^{+}\right)\end{array}$ & $\begin{array}{c}\text { ToLCNDV } \\
\text { Resistance } \\
\text { Reaction }\end{array}$ & $\begin{array}{l}\text { DM Resistance } \\
\text { Reaction }\end{array}$ & $\begin{array}{c}\text { Fruit } \\
\text { Bitterness }\end{array}$ & Fruit Color & $\begin{array}{c}\text { Market } \\
\text { Segment Based } \\
\text { on Fruit Length }\end{array}$ \\
\hline THLA 4 & Bangladesh & $26.3 \pm 0.7$ & $31.3 \pm 3.5$ & Resistant & Susceptible & Non-bitter & Green & Medium \\
\hline THLA 20 & Bangladesh & $26.3 \pm 0.7$ & $25.0 \pm 0.0$ & Resistant & Susceptible & Non-bitter & Green & Medium \\
\hline THLA 31 & Bangladesh & $38.0 \pm 5.6$ & $23.0 \pm 0.0$ & Resistant & Susceptible & Non-bitter & Green & Short \\
\hline THLA 36 & Bangladesh & $39.7 \pm 1.7$ & $25.7 \pm 1.3$ & Resistant & Susceptible & Non-bitter & Green & Short \\
\hline THLA 38 & Bangladesh & $50.3 \pm 0.3$ & $24.0 \pm 2.1$ & Resistant & Moderately resistant & Non-bitter & Green & Short \\
\hline THLA 39 & Bangladesh & $31.7 \pm 2.3$ & $22.3 \pm 0.7$ & Resistant & Resistant & Non-bitter & Green & Short \\
\hline THLA 40 & Bangladesh & $26.3 \pm 4.9$ & $25.7 \pm 0.7$ & Resistant & Susceptible & Non-bitter & Green & Short \\
\hline THLA 41 & Bangladesh & $28.7 \pm 1.7$ & $35.3 \pm 1.8$ & Resistant & Moderately resistant & Non-bitter & Green & Long \\
\hline THLA 45 & Bangladesh & $35.0 \pm 0.6$ & $29.3 \pm 2.3$ & Resistant & Susceptible & Non-bitter & Green & Short \\
\hline THLA 48 & Bangladesh & $28.7 \pm 1.7$ & $34.3 \pm 11.3$ & Resistant & Susceptible & Non-bitter & Green & Short \\
\hline THLA 51 & Bangladesh & $39.3 \pm 2.3$ & $23.7 \pm 0.7$ & Resistant & Moderately resistant & Non-bitter & Green & Short \\
\hline THLA 54 & Bangladesh & $25.0 \pm 3.6$ & $25.7 \pm 0.7$ & Resistant & Susceptible & Non-bitter & Light green & Short \\
\hline THLA 62 & Bangladesh & $25.0 \pm 1.2$ & $23.0 \pm 0.0$ & Resistant & Susceptible & Non-bitter & Green & Short \\
\hline THLA 64 & Bangladesh & $32.0 \pm 2.5$ & $23.0 \pm 0.0$ & Resistant & Susceptible & Non-bitter & Green & Short \\
\hline THLA 67 & Bangladesh & $47.0 \pm 1.5$ & $23.0 \pm 0.0$ & Resistant & Moderately resistant & Non-bitter & Green & Short \\
\hline THLA 70 & Bangladesh & $36.0 \pm 1.2$ & $23.7 \pm 0.7$ & Resistant & Moderately resistant & Non-bitter & Green & Short \\
\hline THLA 70-10 & Bangladesh & $24.3 \pm 1.8$ & $43.7 \pm 0.3$ & Resistant & Susceptible & Non-bitter & Green & Short \\
\hline THLA 71 & Bangladesh & $32.3 \pm 2.7$ & $31.0 \pm 5.0$ & Resistant & Susceptible & Non-bitter & Green & Medium \\
\hline THLA 75 & Bangladesh & $29.7 \pm 3.0$ & $25.0 \pm 1.2$ & Resistant & Susceptible & Non-bitter & Green & Short \\
\hline THLA 80 & Bangladesh & $30.0 \pm 3.0$ & $22.0 \pm 1.0$ & Resistant & Susceptible & Non-bitter & Green & Medium \\
\hline THLA 88 & Bangladesh & $28.7 \pm 2.7$ & $23.0 \pm 0.0$ & Resistant & Moderately resistant & Non-bitter & Green & Short \\
\hline THLA 88-10 & Bangladesh & $28.0 \pm 2.1$ & $24.3 \pm 0.7$ & Resistant & Susceptible & Non-bitter & Green & Medium \\
\hline THLA 89 & Bangladesh & $29.7 \pm 4.2$ & $23.7 \pm 0.7$ & Resistant & Resistant & Non-bitter & Dark green & Medium \\
\hline THLA 94 & Bangladesh & $27.3 \pm 2.6$ & $26.0 \pm 3.0$ & Resistant & Susceptible & Non-bitter & Green & Short \\
\hline THLA 96 & Bangladesh & $25.0 \pm 1.2$ & $23.3 \pm 1.7$ & Resistant & Susceptible & Non-bitter & Green & Short \\
\hline THLA 108 & Bangladesh & $30.3 \pm 2.7$ & $29.3 \pm 2.3$ & Resistant & Susceptible & Non-bitter & Green & Medium \\
\hline THLA 109 & Bangladesh & $39.7 \pm 6.8$ & $23.0 \pm 0.0$ & Resistant & Moderately resistant & Non-bitter & Green & Medium \\
\hline
\end{tabular}


Table 1. Cont.

\begin{tabular}{|c|c|c|c|c|c|c|c|c|}
\hline Entry & Origin & $\begin{array}{c}\text { Days } 50 \% \\
\text { Pistillate } \\
\text { Flowering } \\
\text { (mean } \pm \text { SE) }\end{array}$ & $\begin{array}{c}\text { Days } 50 \% \\
\text { Staminate } \\
\text { Flowering } \\
\left(\text { mean } \pm \mathrm{SE}^{+}\right)\end{array}$ & $\begin{array}{l}\text { ToLCNDV } \\
\text { Resistance } \\
\text { Reaction }\end{array}$ & $\begin{array}{l}\text { DM Resistance } \\
\text { Reaction }\end{array}$ & $\begin{array}{c}\text { Fruit } \\
\text { Bitterness }\end{array}$ & Fruit Color & $\begin{array}{c}\text { Market } \\
\text { Segment Based } \\
\text { on Fruit Length }\end{array}$ \\
\hline THLA 111 & Bangladesh & $23.3 \pm 2.0$ & $23.0 \pm 0.0$ & Resistant & Susceptible & Non-bitter & Green & Medium \\
\hline THLA 114 & Bangladesh & $24.3 \pm 1.8$ & $23.7 \pm 0.7$ & Resistant & Susceptible & Non-bitter & Green & Long \\
\hline THLA 114-9 & Bangladesh & $24.3 \pm 1.8$ & $23.7 \pm 0.7$ & Resistant & Susceptible & Non-bitter & Green & Medium \\
\hline THLA 116 & Bangladesh & $28.3 \pm 3.3$ & $22.7 \pm 0.3$ & Resistant & Susceptible & Non-bitter & Green & Medium \\
\hline THLA 120 & Bangladesh & $35.0 \pm 0.6$ & $23.7 \pm 0.7$ & Resistant & Susceptible & Non-bitter & Green & Short \\
\hline THLA 121 & Bangladesh & $34.7 \pm 0.3$ & $33.0 \pm 3.0$ & Resistant & Susceptible & Non-bitter & Green & Medium \\
\hline THLA $121-10$ & Bangladesh & $38.0 \pm 1.2$ & $32.0 \pm 0.0$ & Resistant & Moderately resistant & Non-bitter & Green & Short \\
\hline THLA 123 & Bangladesh & $36.3 \pm 1.3$ & $24.3 \pm 1.2$ & Resistant & Susceptible & Non-bitter & Green & Short \\
\hline THLA125 & Bangladesh & $42.3 \pm 0.3$ & $20.7 \pm 0.3$ & Resistant & Susceptible & Non-bitter & Green & Short \\
\hline THLA 126 & Bangladesh & $44.3 \pm 1.5$ & $23.7 \pm 0.7$ & Resistant & Susceptible & Non-bitter & Green & Medium \\
\hline THLA 127 & Bangladesh & $44.0 \pm 2.1$ & $21.7 \pm 1.7$ & Resistant & Susceptible & Non-bitter & Green & Medium \\
\hline THLA 128 & Bangladesh & $22.7 \pm 0.3$ & $23.0 \pm 0.0$ & Resistant & Susceptible & Non-bitter & Green & Short \\
\hline THLA 130 & Bangladesh & $44.3 \pm 2.2$ & $22.7 \pm 1.2$ & Resistant & Susceptible & Non-bitter & Green & Short \\
\hline THLA 134 & Bangladesh & $37.3 \pm 6.3$ & $23.7 \pm 0.7$ & Resistant & Susceptible & Non-bitter & Green & Short \\
\hline THLA 136 & Bangladesh & $26.0 \pm 3.0$ & $23.7 \pm 0.7$ & Resistant & Susceptible & Non-bitter & Green & Medium \\
\hline THLA 137 & Bangladesh & $23.7 \pm 0.7$ & $31.3 \pm 3.2$ & Resistant & Susceptible & Non-bitter & Green & Medium \\
\hline THLA 137-9 & Bangladesh & $29.7 \pm 2.3$ & $46.3 \pm 3.7$ & Resistant & Susceptible & Non-bitter & Green & Short \\
\hline THLA 139 & Bangladesh & $37.0 \pm 2.0$ & $32.0 \pm 0.0$ & Resistant & Susceptible & Non-bitter & Green & Short \\
\hline THLA 142 & Bangladesh & $27.7 \pm 3.2$ & $25.0 \pm 0.0$ & Resistant & Resistant & Non-bitter & Green & Short \\
\hline THLA 143 & Bangladesh & $32.0 \pm 2.5$ & $28.7 \pm 1.7$ & Resistant & Susceptible & Non-bitter & Green & Short \\
\hline THLA 145 & Bangladesh & $36.0 \pm 5.5$ & $28.7 \pm 2.7$ & Resistant & Susceptible & Non-bitter & Green & Short \\
\hline THLA 146 & Bangladesh & $30.0 \pm 3.0$ & $32.7 \pm 0.7$ & Resistant & Susceptible & Non-bitter & Green & Short \\
\hline THLA 147 & Bangladesh & $28.0 \pm 2.1$ & $25.7 \pm 0.7$ & Resistant & Susceptible & Non-bitter & Green & Short \\
\hline THLA 149 & Bangladesh & $25.7 \pm 0.7$ & $25.7 \pm 0.7$ & Resistant & Susceptible & Non-bitter & Green & Short \\
\hline THLA 151 & Bangladesh & $33.3 \pm 0.7$ & $21.0 \pm 0.0$ & Resistant & Resistant & Non-bitter & Green & Medium \\
\hline THLA 152 & Bangladesh & $31.3 \pm 4.7$ & $23.7 \pm 1.5$ & Resistant & Moderately resistant & Non-bitter & Green & Short \\
\hline THLA 154 & Bangladesh & $25.7 \pm 3.5$ & $24.3 \pm 0.9$ & Resistant & Susceptible & Non-bitter & Green & Long \\
\hline
\end{tabular}


Table 1. Cont.

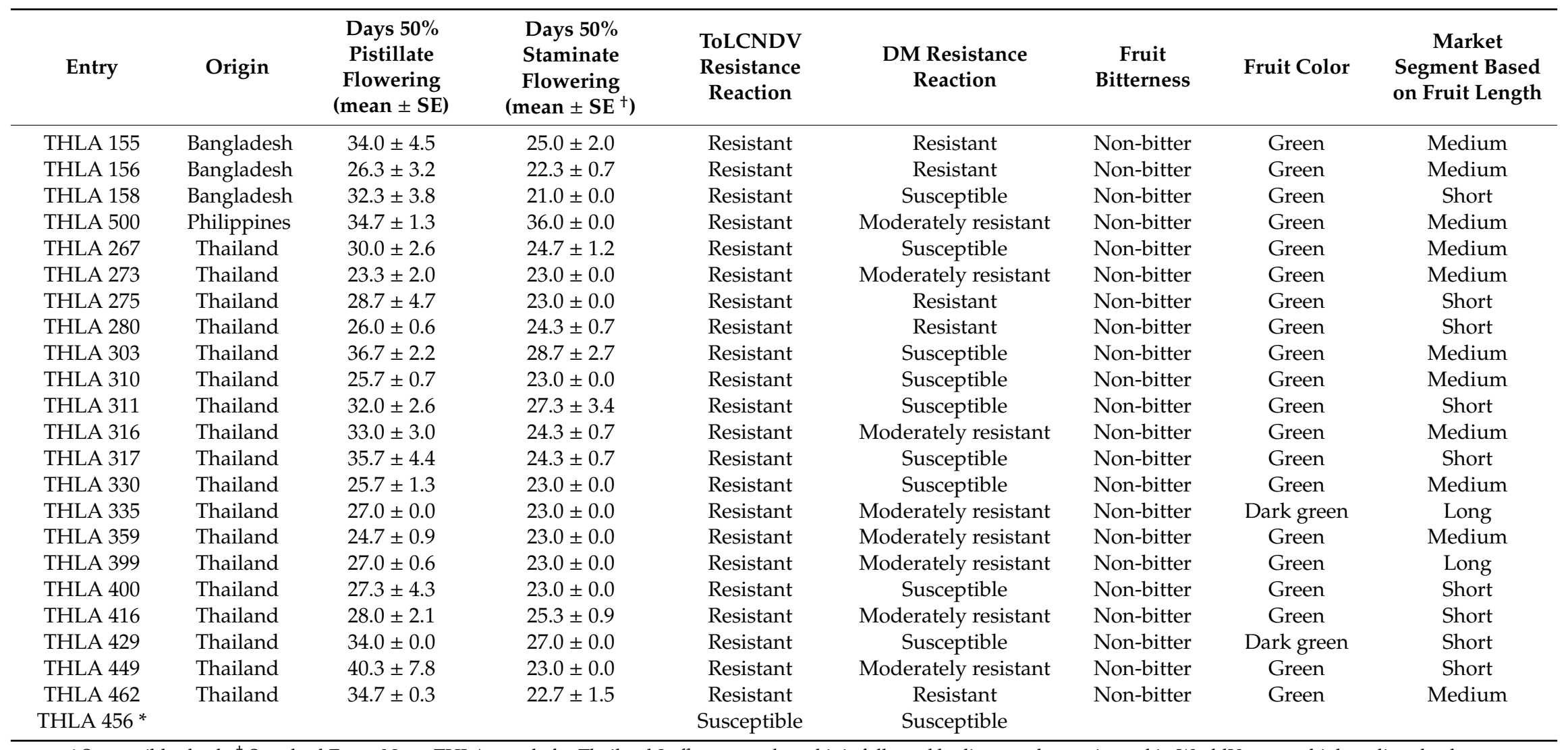

${ }^{*}$ Susceptible check; ${ }^{+}$Standard Error. Note: THLA stands for Thailand Luffa acutangula and it is followed by line number registered in WorldVeg cucurbit breeding database. 
Table 2. Summary of disease reaction of lines of sponge gourd (Luffa cylindrica) to ToLCNDV and DM, days to 50\% pistillate and staminate flowering, and fruit traits of lines field evaluated in 2020 at the WorldVeg, Thailand.

\begin{tabular}{|c|c|c|c|c|c|c|c|c|}
\hline Entry & Origin & $\begin{array}{c}\text { Days } 50 \% \text { Pistillate } \\
\text { Flowering (Mean } \pm \text { SE) }\end{array}$ & $\begin{array}{c}\text { Days } 50 \% \text { Staminate } \\
\text { Flowering (Mean } \pm \text { SE) }\end{array}$ & $\begin{array}{c}\text { ToLCNDV } \\
\text { Resistance } \\
\text { Reaction }\end{array}$ & $\begin{array}{l}\text { DM Resistance } \\
\text { Reaction }\end{array}$ & $\begin{array}{c}\text { Fruit } \\
\text { Bitterness }\end{array}$ & $\begin{array}{l}\text { Fruit Length } \\
\text { Segment }\end{array}$ & $\begin{array}{c}\text { Market } \\
\text { Segment Based } \\
\text { on Fruit Color }\end{array}$ \\
\hline THLC 1 & Bangladesh & $35.3 \pm 0.9$ & $35.3 \pm 0.7$ & Resistant & Moderately resistant & Non-bitter & Long & Light green \\
\hline THLC 3 & Bangladesh & $43.7 \pm 0.9$ & $44.3 \pm 1.2$ & Resistant & Resistant & Non-bitter & Medium & Light green \\
\hline THLC 43 & Bangladesh & $29.7 \pm 2.2$ & $34.7 \pm 0.7$ & Resistant & Resistant & Non-bitter & Medium & Light green \\
\hline THLC 43-9 & Bangladesh & $27.0 \pm 0.0$ & $34.0 \pm 0.0$ & Resistant & Moderately resistant & Non-bitter & Long & Green \\
\hline THLC 45-7-2 & Bangladesh & $33.7 \pm 0.3$ & $34.0 \pm 0.0$ & Resistant & Resistant & Non-bitter & Medium & Light green \\
\hline THLC $45-7-5$ & Bangladesh & $33.7 \pm 0.3$ & $34.0 \pm 0.0$ & Resistant & Resistant & Non-bitter & Medium & Green \\
\hline THLC 45-8 & Bangladesh & $36.3 \pm 0.7$ & $40.0 \pm 2.0$ & Resistant & Resistant & Non-bitter & Medium & Light green \\
\hline THLC 46 & Bangladesh & $32.0 \pm 2.0$ & $34.3 \pm 0.3$ & Resistant & Moderately resistant & Non-bitter & Long & Light green \\
\hline THLC 46-5 & Bangladesh & $26.3 \pm 0.3$ & $34.0 \pm 0.0$ & Resistant & Susceptible & Non-bitter & Short & Green \\
\hline THLC 56 & Bangladesh & $30.3 \pm 1.9$ & $31.7 \pm 2.3$ & Resistant & Moderately resistant & Non-bitter & Medium & Light green \\
\hline THLC 57 & Bangladesh & $33.7 \pm 0.3$ & $35.3 \pm 1.3$ & Resistant & Resistant & Non-bitter & Medium & Dark green \\
\hline THLC 60 & Bangladesh & $27.7 \pm 1.9$ & $31.3 \pm 2.7$ & Resistant & Moderately resistant & Non-bitter & Medium & Dark green \\
\hline THLC 62 & Bangladesh & $26.3 \pm 0.3$ & $28.0 \pm 3.1$ & Resistant & Susceptible & Non-bitter & Long & Green \\
\hline THLC 70 & Bangladesh & $25.0 \pm 1.2$ & $25.0 \pm 0.6$ & Resistant & Susceptible & Non-bitter & Long & Dark green \\
\hline THLC 73 & Bangladesh & $24.0 \pm 0.0$ & $34.0 \pm 0.0$ & Resistant & Moderately resistant & Non-bitter & Long & Green \\
\hline THLC 83 & Bangladesh & $26.7 \pm 0.3$ & $31.7 \pm 2.3$ & Resistant & Moderately resistant & Non-bitter & Short & Dark green \\
\hline THLC 87 & Bangladesh & $27.3 \pm 0.3$ & $35.0 \pm 0.6$ & Resistant & Resistant & Non-bitter & Medium & Green \\
\hline THLC 96 & Bangladesh & $28.7 \pm 2.7$ & $32.3 \pm 1.7$ & Resistant & Moderately resistant & Non-bitter & Medium & Green \\
\hline THLC 97 & Bangladesh & $28.0 \pm 1.0$ & $28.7 \pm 0.3$ & Resistant & Susceptible & Non-bitter & Long & Dark green \\
\hline THLC 99 & Bangladesh & $24.3 \pm 1.5$ & $26.3 \pm 1.8$ & Resistant & Susceptible & Non-bitter & Medium & Light green \\
\hline THLC 99-4 & Bangladesh & $24.3 \pm 1.5$ & $26.3 \pm 1.8$ & Resistant & Resistant & Non-bitter & Medium & Dark green \\
\hline THLC 104 & Bangladesh & $27.3 \pm 0.3$ & $29.0 \pm 0.0$ & Resistant & Resistant & Non-bitter & Medium & Green \\
\hline THLC 113 & Bangladesh & $25.7 \pm 1.3$ & $30.7 \pm 1.7$ & Resistant & Moderately resistant & Non-bitter & Long & Green \\
\hline THLC 115 & Bangladesh & $24.7 \pm 0.7$ & $24.3 \pm 1.3$ & Resistant & Moderately resistant & Non-bitter & Medium & Dark green \\
\hline THLC 119 & Bangladesh & $27.0 \pm 1.5$ & $32.3 \pm 1.7$ & Resistant & Susceptible & Non-bitter & Medium & Green \\
\hline THLC 120 & Bangladesh & $26.3 \pm 0.7$ & $32.3 \pm 1.7$ & Resistant & Susceptible & Non-bitter & Medium & Light green \\
\hline THLC 124 & Bangladesh & $34.3 \pm 0.3$ & $35.0 \pm 0.6$ & Resistant & Moderately resistant & Non-bitter & Long & Light green \\
\hline THLC 125 & Bangladesh & $34.0 \pm 0.6$ & $39.7 \pm 2.8$ & Resistant & Moderately resistant & Non-bitter & Long & Light green \\
\hline THLC 134 & Bangladesh & $28.3 \pm 1.2$ & $34.0 \pm 0.0$ & Resistant & Susceptible & Non-bitter & Medium & Dark green \\
\hline THLC 134-6 & Bangladesh & $35.7 \pm 1.7$ & $32.7 \pm 1.3$ & Resistant & Moderately resistant & Non-bitter & Medium & Light green \\
\hline THLC 136 & Bangladesh & $27.7 \pm 1.2$ & $34.7 \pm 0.7$ & Resistant & Moderately resistant & Non-bitter & Medium & Dark green \\
\hline THLC 138 & Bangladesh & $35.0 \pm 1.2$ & $36.7 \pm 1.3$ & Resistant & Moderately resistant & Non-bitter & Medium & Green \\
\hline
\end{tabular}


Table 2. Cont.

\begin{tabular}{|c|c|c|c|c|c|c|c|c|}
\hline Entry & Origin & $\begin{array}{c}\text { Days } 50 \% \text { Pistillate } \\
\text { Flowering (Mean } \pm \text { SE) }\end{array}$ & $\begin{array}{l}\text { Days } 50 \% \text { Staminate } \\
\text { Flowering (Mean } \pm \text { SE) }\end{array}$ & $\begin{array}{c}\text { ToLCNDV } \\
\text { Resistance } \\
\text { Reaction }\end{array}$ & $\begin{array}{l}\text { DM Resistance } \\
\text { Reaction }\end{array}$ & $\begin{array}{c}\text { Fruit } \\
\text { Bitterness }\end{array}$ & $\begin{array}{l}\text { Fruit Length } \\
\text { Segment }\end{array}$ & $\begin{array}{c}\text { Market } \\
\text { Segment Based } \\
\text { on Fruit Color }\end{array}$ \\
\hline THLC 139 & Bangladesh & $29.0 \pm 1.5$ & $34.0 \pm 0.0$ & Resistant & Moderately resistant & Non-bitter & Long & Green \\
\hline THLC 141 & Bangladesh & $25.0 \pm 1.5$ & $30.7 \pm 1.7$ & Resistant & Susceptible & Non-bitter & Medium & Light green \\
\hline THLC 143 & Bangladesh & $32.3 \pm 2.2$ & $34.0 \pm 0.0$ & Resistant & Moderately resistant & Non-bitter & Medium & Light green \\
\hline THLC 147 & Bangladesh & $28.0 \pm 0.0$ & $34.0 \pm 0.0$ & Resistant & Moderately resistant & Non-bitter & Medium & Light green \\
\hline THLC 152 & Bangladesh & $29.7 \pm 1.8$ & $35.3 \pm 1.3$ & Resistant & Moderately resistant & Non-bitter & Medium & Green \\
\hline THLC 153 & Bangladesh & $30.7 \pm 1.2$ & $35.0 \pm 1.0$ & Resistant & Moderately resistant & Non-bitter & Medium & Light green \\
\hline THLC 156 & Bangladesh & $34.0 \pm 0.0$ & $34.0 \pm 0.0$ & Resistant & Moderately resistant & Non-bitter & Medium & Dark green \\
\hline THLC 158 & Bangladesh & $29.3 \pm 2.3$ & $32.3 \pm 1.7$ & Resistant & Moderately resistant & Non-bitter & Short & Green \\
\hline THLC 168 & Bangladesh & $36.0 \pm 1.5$ & $36.3 \pm 1.5$ & Resistant & Susceptible & Non-bitter & Medium & Green \\
\hline THLC 169 & Bangladesh & $32.3 \pm 1.7$ & $34.7 \pm 0.7$ & Resistant & Susceptible & Non-bitter & Medium & Dark green \\
\hline THLC 170 & Bangladesh & $45.5 \pm 2.9$ & $43.0 \pm 2.9$ & Resistant & Resistant & Non-bitter & Medium & Green \\
\hline THLC 181 & Bangladesh & $30.7 \pm 3.3$ & $34.0 \pm 0.0$ & Resistant & Moderately resistant & Non-bitter & Long & Light green \\
\hline THLC 190 & Bangladesh & $37.0 \pm 0.6$ & $34.7 \pm 0.7$ & Resistant & Resistant & Non-bitter & Long & Light green \\
\hline THLC 193 & Bangladesh & $34.0 \pm 0.0$ & $35.0 \pm 0.6$ & Resistant & Resistant & Non-bitter & Short & Light green \\
\hline THLC 195 & Bangladesh & $37.7 \pm 0.7$ & $35.7 \pm 1.7$ & Resistant & Moderately resistant & Non-bitter & Long & Light green \\
\hline THLC 197 & Bangladesh & $29.3 \pm 0.7$ & $28.3 \pm 0.7$ & Resistant & Moderately resistant & Non-bitter & Long & Dark green \\
\hline THLC 198 & Bangladesh & $38.3 \pm 1.8$ & $38.3 \pm 1.8$ & Resistant & Moderately resistant & Non-bitter & Long & Dark green \\
\hline THLC 203 & Bangladesh & $41.3 \pm 0.3$ & $34.0 \pm 0.0$ & Resistant & Moderately resistant & Non-bitter & Long & Light green \\
\hline THLC 204 & Bangladesh & $38.3 \pm 2.3$ & $36.3 \pm 1.5$ & Resistant & Susceptible & Non-bitter & Long & Light green \\
\hline THLC 843 & Bangladesh & $34.3 \pm 0.3$ & $35.0 \pm 0.6$ & Resistant & Resistant & Non-bitter & Long & Dark green \\
\hline THLC 213 & Cambodia & $35.7 \pm 0.9$ & $35.3 \pm 1.3$ & Resistant & Resistant & Non-bitter & Long & Green \\
\hline THLC 765 & Indonesia & $33.3 \pm 0.3$ & $38.0 \pm 2.3$ & Resistant & Resistant & Non-bitter & Long & Green \\
\hline THLC 387 & Thailand & $35.7 \pm 1.7$ & $35.3 \pm 1.3$ & Resistant & Moderately resistant & Non-bitter & Long & Green \\
\hline THLC 406 & Thailand & $36.7 \pm 2.7$ & $35.3 \pm 0.7$ & Resistant & Resistant & Non-bitter & Long & Green \\
\hline THLC 414 & Thailand & $34.0 \pm 0.0$ & $34.0 \pm 3.1$ & Resistant & Resistant & Non-bitter & Long & Dark green \\
\hline THLC 424 & Thailand & $36.3 \pm 3.8$ & $34.0 \pm 0.0$ & Resistant & Resistant & Non-bitter & Medium & Green \\
\hline THLC 455 & Thailand & $30.0 \pm 1.5$ & $36.0 \pm 1.0$ & Resistant & Resistant & Non-bitter & Long & Green \\
\hline THLC 459 & Thailand & $50.3 \pm 1.7$ & $36.3 \pm 1.2$ & Resistant & Resistant & Non-bitter & Short & Light green \\
\hline THLC 463 & Thailand & $27.0 \pm 0.6$ & $32.3 \pm 1.7$ & Resistant & Moderately resistant & Non-bitter & Long & Dark green \\
\hline THLC 833 & Thailand & $37.7 \pm 2.6$ & $34.7 \pm 0.7$ & Resistant & Resistant & Non-bitter & Short & Green \\
\hline THLC 781 & Vietnam & $39.7 \pm 3.4$ & $34.0 \pm 0.0$ & Resistant & Resistant & Non-bitter & Long & Green \\
\hline THLC 798 & Vietnam & $32.3 \pm 0.3$ & $34.0 \pm 0.0$ & Resistant & Resistant & Non-bitter & Long & Green \\
\hline THLC 799 & Vietnam & $35.3 \pm 1.2$ & $37.3 \pm 2.0$ & Resistant & Susceptible & Non-bitter & Medium & Green \\
\hline THLC $684^{*}$ & & & & Susceptible & Susceptible & & & \\
\hline
\end{tabular}

* Susceptible check. Note: THLC stands for Thailand Luffa cylindrica and it is followed by line number registered in WorldVeg cucurbit breeding database. 


\section{Results}

\subsection{Evaluation of Luffa acutangula Lines}

"THLA 456" (susceptible check) was susceptible to ToLCNDV (mean rating $=2)$ and DM $(>41 \%$ symptomatic leaf area). Eighty-two lines of Luffa acutangula evaluated in this study were resistant to ToLCNDV (mean rating $=0$; Table 1$)$. A majority $(62 / 76.8 \%)$ of the ToLCNDV-resistant lines were derived from landraces originating in Bangladesh, while 20 (24.1\%) of the resistant lines were developed from the landraces originating in Thailand. A single virus-resistant line originated in the Philippines. These ToLCNDV-resistant lines were categorized into three commercial market segments based on fruit length, namely: short (47/57.3\%), medium (30/36.6\%), and long (5/6.1\%; Figure 1$)$. The fruits of these lines were non-bitter. Three distinct fruit skin colors were observed in the ToLCNDV-resistant lines, namely: green (75/91.4\%), light green (1/1.2\%), and dark green (6/7.4\%). Days to $50 \%$ pistillate and staminate flowering (after transplanting) ranged from 21-50 and 21-46, respectively, among these ToLCNDV-resistant lines. The earliest ( $<25$ days to $50 \%$ flowering) pistillate flowering lines originated in Bangladesh.

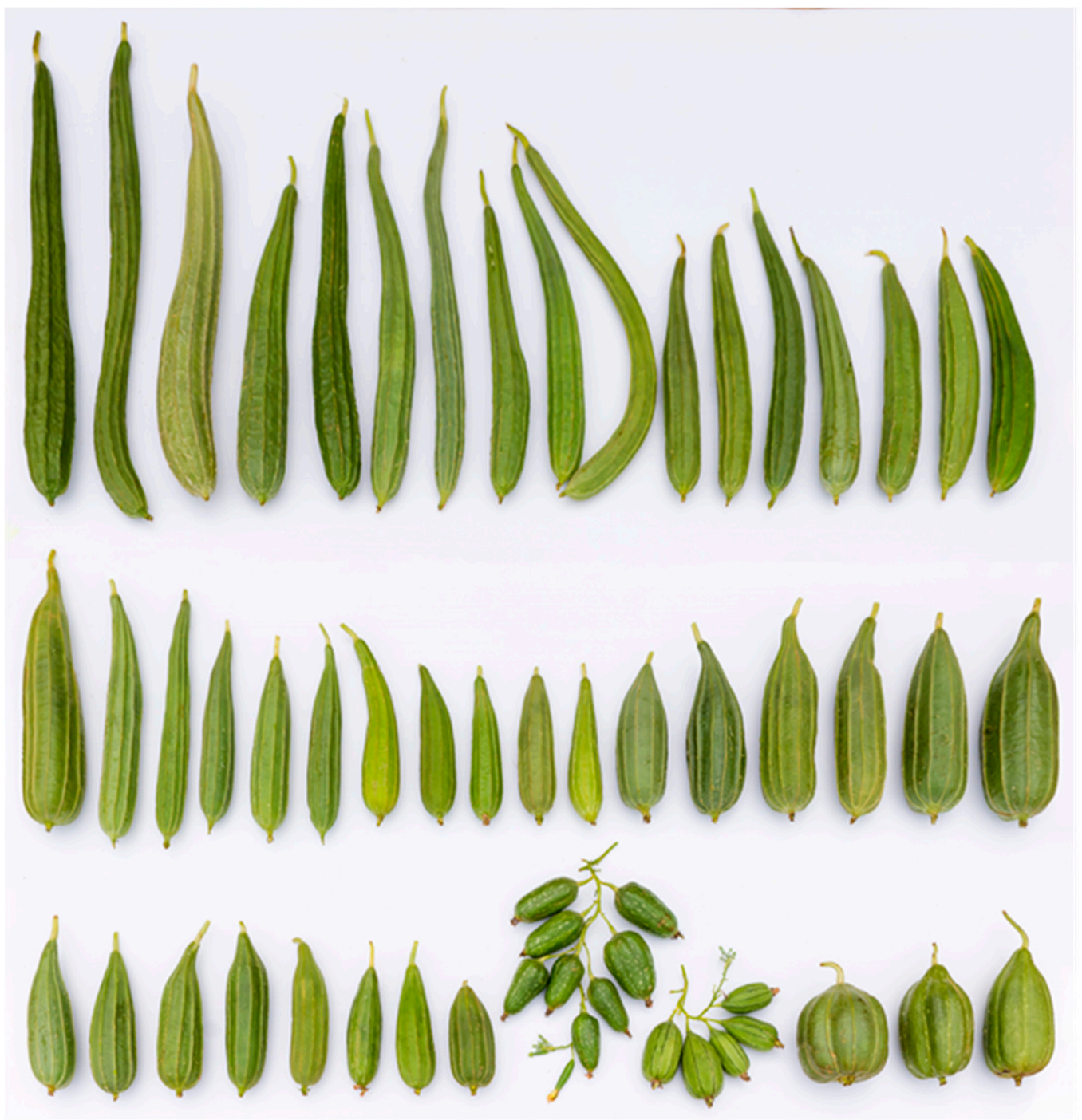

Figure 1. Fruit color and length variability among ridge gourd breeding lines.

Resistance to ToLCNDV in ridge gourd is conditioned by a single dominant gene [37]. A dark green fruit color is dominant over a green and light green color, whereas a green fruit color is 
dominant over light green [37]. The identified ToLCNDV-resistant lines provide ample opportunity for the development of early maturing, virus-resistant $F_{1}$ hybrid cultivars of various market segments possessing the dark green and green fruit colors preferred by consumers.

Six and three DM-resistant lines originated in Bangladesh and Thailand, respectively. All were non-bitter. The fruit colors of eight and one DM-resistant lines were green and dark green, respectively. Ridge gourd cultivars resistant to DM are not currently available. Hence, this germplasm is a potential source of resistance for DM in ridge gourd and is of importance to public and private sector breeding programs in Asia.

\subsection{Evaluation of Luffa cylindrica Lines}

The sponge gourd susceptible check line "THLC 684" was susceptible to both ToLCNDV (mean rating $=2$ ) and DM ( $>41 \%$ symptomatic leaf area). All 65 breeding lines were resistant to ToLCNDV (mean rating $=0$; Table 2). These lines originated in Bangladesh $(52 / 80 \%)$, Thailand $(8 / 12.3 \%)$, Vietnam (3/4.6\%), Cambodia $(1 / 1.5 \%)$, and Indonesia $(1 / 1.5 \%)$. Three distinct fruit skin colors were observed, namely: green $(26 / 40 \%)$, light green $(23 / 35.4 \%)$, and dark green $(16 / 24.6 \%)$. The following three categories of fruit length were observed: short (6/9.2\%), medium (31/47.7\%), and long $(28 / 43.1 \%$; Figure 2). The fruit of all of the lines were non-bitter. Days to $50 \%$ pistillate and staminate flowering (after transplanting) ranged from $24-50$ and 24-44), respectively. All of the earliest ( $<25$ days to $50 \%$ flowering) pistillate flowering lines originated in Bangladesh.

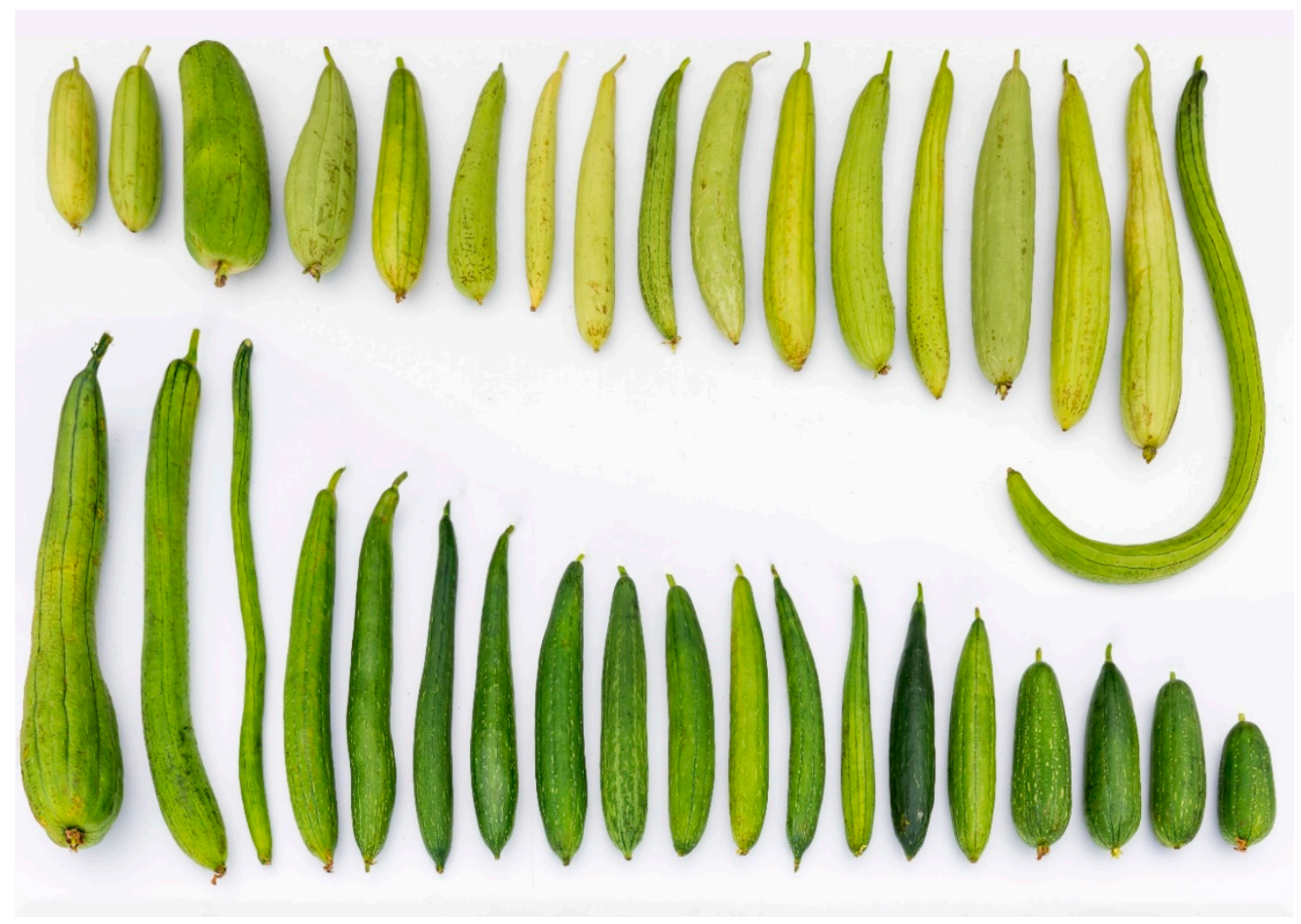

Figure 2. Fruit color and length variability among sponge gourd breeding lines.

Resistance to ToLCNDV in sponge gourd was determined to be governed by a single dominant gene [38]. Commercial sponge gourd cultivars resistant to ToLCNDV are currently unavailable in the global seed market. Therefore, the ToLCNDV-resistant lines described here provide an opportunity for the development of virus resistant $\mathrm{F}_{1}$ hybrid cultivars.

Although none of the sponge gourd lines were immune to DM, 23 lines were resistant (1-10\% symptomatic leaf area). Resistant lines (36) originated in Bangladesh and Southeast Asia (22). 
Sponge gourd cultivars resistant to DM are unavailable in all sponge gourd market segments. Hence, this germplasm could be of use in the development of DM-resistant cultivars, so as to fill the various market segments.

\section{Discussion}

ToLCNDV infecting cucurbits results in major economic losses among cucurbits, including loofah, in Asia. The disease can result in 100\% crop loss under epidemic conditions [39-41]. A sponge gourd accession "DSG-6" from India was previously reported to be resistant to ToLCNDV. That resistance was determined to be governed by a single dominant gene [38]. However, the seed industry has not been able to exploit that resistance to develop $F_{1}$ hybrids, as the resistance failed to persist during multi-location trials across India [37]. In addition, the fruits of "DSG-6" are dark green with superficial stripes. However, the sponge gourd market in India, Pakistan, and Bangladesh is dominated by cultivars producing green or light green fruit without stripes. "DSG-6" originated in West Bengal (India). Interestingly, the majority of the sponge gourd lines found to be resistant to ToLCNDV in this study originated in Bangladesh, which was part of the West Bengal state of India before the partition of British India in 1947. The Indo-Burma region and India (including Bangladesh) are considered the primary centers of diversity of sponge gourd and ridge gourd, respectively [42], although the exact area of domestication of sponge and ridge gourds is disputed [43]. Sponge gourd material from Bangladesh also provided the earliest pistillate flowering lines. Ridge gourd lines resistant to ToLCNDV have not been previously reported, although Premchand et al. [44] evaluated germplasm from India for resistance to this begomovirus. The first commercial ridge gourd $\mathrm{F}_{1}$ hybrid cultivar "Arti", resistant to ToLCNDV, was released in India by VNR Seeds Private Limited in 2008 [37]. However, the resistance proved to be unstable across locations in India.

Downy mildew was observed in sponge gourd fields of commercial farmers in the Odisha state of India in 2014 [24]. Sources of DM resistance in ridge gourd and sponge gourd have not been reported. The current study has identified sources of DM resistance in the short and medium fruit length market segments of ridge gourd, and in the short, medium, and long fruit length market segments of sponge gourd. These include green, light green, and dark green fruit skin colors. These resistant lines provide a basis for the development of new DM-resistant elite ridge gourd and sponge gourd $\mathrm{F}_{1}$ hybrids. Multiple genes for DM resistance have been reported in cucumber and melon $[45,46]$. Information on the genetic mechanisms of resistance to DM in loofah is lacking in the literature.

ToLCNDV- and DM-resistant lines will be showcased during the WorldVeg's Loofah Open Field Day in 2021. These lines are being further evaluated for ToLCNDV and DM in multi-location trials across Asia, in partnership with seed companies and public institutes. SNP-based genetic diversity analysis of these lines, and a study of their relationship with commercial hybrid cultivars currently popular with the farmers in Asia, is in progress.

\section{Conclusions}

Ridge gourd and sponge gourd fruit contribute to human nutrition, and their production and sale provides a livelihood for resource-poor farmers in Asia. These cucurbits are also important members of home, school, and community gardens in the tropics. ToLCNDV and DM are the major production constraints faced by growers in Asia, and commercial cultivars resistant to both these economic diseases are currently unavailable. The adoption of disease-resistant cultivars is an economically sound approach for disease management. The very few currently available hybrid cultivars of loofah are popular with farmers. However, these cultivars have a narrow genetic base. The current work utilized the genetically diverse landraces of loofah maintained in the WorldVeg genebank to identify and develop loofah inbred lines resistant to ToLCNDV and DM. This assortment of genetically broad-based breeding lines includes those producing fruits displaying horticultural traits associated with various commercial segments of loofah. Hence, these lines provide an opportunity for the global loofah seed industry to develop new $\mathrm{F}_{1}$ hybrids resistant to ToLCNDV and DM to meet the needs of both 
growers and consumers. The majority of the loofah lines resistant to ToLCNDV and DM originated in Bangladesh. Thus, future loofah germplasm collection and conservation efforts should focus on the loofah landraces present in the different agro-climatic regions of that country.

Author Contributions: N.P.S.D. and M.A.T.M. conceived the study and designed the research. S.P., M.N., and S.L. performed the experiments. N.P.S.D., S.P., M.N., and S.L. evaluated the germplasm in the field. M.N. and S.L. analyzed the data and prepared the tables. N.P.S.D., M.A.T.M., and R.L.J. wrote the paper. All authors have read and agreed to the published version of the manuscript.

Funding: This research received external funding from twenty-five collaborative seed companies (World Vegetable Center project numbers: 10000274, 10000292, 10000365).

Acknowledgments: Funding for this research was provided by strategic long-term donors to the World Vegetable Center, namely: Republic of China (Taiwan), UK aid from the U.K. government, United States Agency for International Development (USAID), Australian Centre for International Agricultural Research (ACIAR), Germany, Thailand, Philippines, Korea, and Japan.

Conflicts of Interest: The authors declare no conflict of interest.

\section{References}

1. Dhillon, N.P.S.; Sanguansil, S.; Singh, S.P.; Masud, M.A.T.; Kumar, P.; Bharathi, L.K.; Yetisir, H.; Huang, R.; Canh, D.X.; McCreight, J.D. Gourds: Bitter, Bottle, Wax, Snake, Sponge and Ridge. In Genetics and Genomics of Cucurbitaceae; Grumet, R., Katzir, N., Garcia-Mas, J., Eds.; Springer International Publishing AG: Cham, Switzerland, 2017; pp. 155-172.

2. US Department of Agriculture (USDA). National Nutrient Database for Standard Reference. Available online: http://www.ars.usda.gov/Services/docs.htm?docid=8964 (accessed on 1 March 2020).

3. Chawda, V.; (VNR Seeds Private Limited, Raipur, Chhattisgarh, India); Anil, M.; (Acsen HyVeg Private Limited, Bangalore, Karnataka, India); Hegde, G.; (Noble Seeds Private Limited, Bangalore, Karnataka, India). Personal communication, 2020.

4. Masud, A. (Bangladesh Agricultural Research Institute, Gazipur, Bangladesh). Personal communication, 2020.

5. Lin, M.L. (World Vegetable Center, Shannhua, Taiwan). Personal communication, 2020.

6. Srivastava, K.M.; Hallan, V.; Raizada, R.K.; Chandra, G.; Singh, B.P.; Sane, P.V. Molecular cloning of Indian tomato leaf curl virus genome following a simple method of concentrating the supercoiled replicative form of viral DNA. J. Virol. Methods 1995, 51, 297-304. [CrossRef]

7. Juarez, M.; Tovar, R.; Fiallo-Olive, E.; Aranda, M.A.; Gosalvez, B.; Castillo, P.; Moriones, E.; Navas-Castillo, J. First detection of tomato leaf curl New Delhi virus infecting zucchini in Spain. Plant Dis. 2014, 98, 857-858. [CrossRef] [PubMed]

8. Mnari-Hattab, M.; Zammouri, S.; Belkadhi, M.S.; Bellen Dona, D.; Ben Nahia, E.; Hajlaoui, M.R. First report of tomato leaf curl New Delhi virus infecting cucurbits in Tunisia. New Dis. Rep. 2015, 31, 21. [CrossRef]

9. Panno, S.; Iacono, G.; Davino, M.; Marchione, S.; Zappardo, V.; Bella, P.; Tomassoli, L.; Accotto, G.P.; Davino, S. First report of tomato leaf curl New Delhi virus affecting zucchini squash in an important horticultural area of southern Italy. New Dis. Rep. 2016, 33, 6. [CrossRef]

10. Sifres, A.; Saez, C.; Ferriol, M.; Selmani, E.; Riado, J.; Pico, B.; Lopez, C. First report of tomato leaf curl New Delhi virus infecting zucchini in Morocco. Plant Dis. 2018, 102, 1045. [CrossRef]

11. Orfanidou, C.G.; Malandraki, I.; Beris, D.; Kektsidou, O.; Vassilakos, N.; Varveri, C.; Katis, N.I.; Maliogka, V.I. First report of tomato leaf curl New Delhi virus in zucchini crops in Greece. J. Plant. Pathol. 2019, 101, 799. [CrossRef]

12. Kheireddine, A.; Sifres, A.; Saez, C.; Pico, B.; López, C. First report of tomato leaf curl New Delhi virus infecting cucurbit plants in Algeria. Plant Dis. 2019, 103, 3291. [CrossRef]

13. European and Mediterranean Plant Protection Organization. Available online: https://www.eppo.int/ QUARANTINE/Alert_List/viruses/ToLCNDV.htm (accessed on 20 November 2019).

14. Sohrab, S.S.; Mandal, B.; Pant, R.P.; Varma, A. First report of association of tomato leaf curl virus-New Delhi with yellow mosaic disease of Luffa cylindrica in India. Plant Dis. 2003, 87, 1148. [CrossRef] [PubMed] 
15. Ito, T.; Sharma, P.; Kittipakorn, K.; Ikegami, M. Complete nucleotide sequence of a new isolate of tomato leaf curl New Delhi virus infecting cucumber, bottle gourd and muskmelon in Thailand. Arch. Virol. 2008, 153, 611-613. [CrossRef]

16. Singh, A.K.; Mishra, K.K.; Chattopadhyay, B.; Chakraborty, S. Biological and molecular characterization of a begomovirus associated with yellow mosaic vein mosaic disease of pumpkin from Northern India. Virus Genes 2009, 39, 359-370. [CrossRef]

17. Chang, H.H.; Ku, H.M.; Tsai, W.S.; Chien, R.C.; Jan, F.J. Identification and characterization of a mechanical transmissible begomovirus causing leaf curl on oriental melon. Eur. J. Plant Pathol. 2010, 127, 219-228. [CrossRef]

18. Roy, A.; Spoorthi, P.; Panwar, G.; Bag, M.K.; Prasad, T.V.; Kumar, G.; Gangopadhaya, K.K.; Dutta, M. Molecular evidence for occurrence of tomato leaf curl New Delhi virus in ash gourd (Benincasa hispida) germplasm showing a severe yellow stunt disease in India. Indian J. Virol. 2013, 24, 74-77. [CrossRef] [PubMed]

19. Cohen, Y.; Rubin, A.E.; Galperin, M.; Ploch, S.; Runge, F.; Thines, M. Seed transmission of Pseudoperonospora cubensis. PLoS ONE 2014, 9, e109766. [CrossRef] [PubMed]

20. Colucci, S.J.; Holmes, G.J. Downy mildew of cucurbits. Plant. Health Instr. 2010. [CrossRef]

21. Palti, J.; Cohen, Y. Downy mildew of cucurbits (Pseudoperonospora cubensis). The fungus and its hosts, distribution, epidemiology and control. Phytoparasitica 1980, 8, 109-147. [CrossRef]

22. Lebeda, A. Screening of wild Cucumis species against downy mildew (Pseudoperonospora cubensis) isolates from cucumbers. Phytoparasitica 1992, 20, 203-210. [CrossRef]

23. Lebeda, A.; Widrlechner, M.P. A set of cucurbitaceae taxa for differentiation of Pseudoperonospora cubensis pathotypes. J. Plant Dis. Prot. 2003, 110, 337-349.

24. Nayak, A.K.; Bandamaravuri, K.B. First report of downy mildew caused by Pseudoperonospora cubensis on Luffa cylindrica in India. J. Plant Pathol. 2018, 101. [CrossRef]

25. Cohen, Y. The combined effects of temperature, leaf wetness and inoculum concentration on infection of cucumbers with Pseudoperonospora cubensis. Can. J. Bot. 1977, 55, 1478-1487. [CrossRef]

26. Palti, J. The significance of pronounced divergences in the distribution of Pseudoperonospora cubensis on its crop hosts. Phytoparasitica 1974, 2, 109-115. [CrossRef]

27. Bains, S.S.; Jhooty, J.S. Host-range and possibility of pathological races in Pseudoperonospora cubensis-Cause of downy mildew of muskmelon. Indian Phtopathol. 1976, 29, 214-216.

28. Inaba, T.; Morinaka, T.; Hamaya, E. Physiological races of Pseudoperonospora cubensis isolated from cucumber and muskmelon in Japan. Bull. Natl. Inst. Agro-Environ. Sci. 1986, 2, 35-43.

29. Angelov, D.; Krasteva, L. Dominant inheritance of downy mildew resistance in melons. Acta Hortic. 2000, 510, 273-276. [CrossRef]

30. Shetty, N.V.; Wehner, T.C.; Thomas, C.E.; Doruchowski, R.W.; Shetty, V.K.P. Evidence for downy mildew races in cucumber tested in Asia, Europe and North America. Sci. Hortic. 2002, 94, 231-239. [CrossRef]

31. Thomas, C.E.; Inaba, T.; Cohen, Y. Physiological specialization in Pseudoperonospora cubensis. Phytopathology 1987, 77, 1621-1624. [CrossRef]

32. Cohen, Y.; Meron, I.; Mor, N.; Zuriel, S. A new pathotype of Pseudoperonospora cubensis causing downy mildew in Israel. Phytoparasitica 2003, 31, 458-466. [CrossRef]

33. Lebeda, A.; Urban, J. Disease impact and pathogenicity variation in Czech populations of Pseudoperonospora cubensis. In Progress in Cucurbit Genetics and Breeding Research, Proceedings of the Cucurbitaceae 2004, the 8th EUCARPIA Meeting on Cucurbit Genetics and Breeding, Olomouc, Czech Republic, 12-17 July 2004; Lebeda, A., Paris, H.S., Eds.; Palacky University in Olomouc: Olomouc, Czech Republic, 2004; pp. 267-273.

34. Doyle, J.J.; Doyle, J.L. Isolation of plant DNA from fresh tissue. Focus 1983, 12, 13-15.

35. Jenkins, S.F.; Wehner, T.C. A system for the measurement of foliar diseases in cucumbers. Cucurbit Genet. Coop. Rep. 1983, 6, 10-12.

36. Waterhouse, G.M.; Brothers, M.P. The taxonomy of Pseudoperonospora. Mycol. Pap. 1981, 148, 1-28.

37. Chawda, V.; (VNR Seeds Private Limited, Raipur, Chhattisgarh, India). Personal communication, 2020.

38. Islam, S.; Munshi, A.D.; Mandal, B.; Kumar, R.; Behera, T.K. Genetics of resistance in Luffa cylindrica Roem. against Tomato leaf curl New Delhi virus. Euphytica 2010, 174, 83-89. [CrossRef]

39. Singh, R.P.; Mohan, J.; Singh, D.P. Symptomatology and distribution of ridge gourd mosaic virus. Agric. Sci. Digest. 2001, 21, 149-152. 
40. Nagendran, K.; Mohankumar, S.; Aravintharaj, R.; Balaji, C.G.; Manoranjitham, S.K.; Singh, A.K.; Rai, A.B.; Singh, B.; Karthikeyan, G. The occurrence and distribution of major viruses infecting cucurbits in Tamil Nadu state, India. Crop. Protect. 2017, 99, 10-16. [CrossRef]

41. Kumar, R.; Esakky, R.; Acharya, S. Molecular evidence of Tomato leaf curl New Delhi virus infecting cucurbits in several states in India. Arch. Phytopath. Plant. Prot. 2019, 52, 895-905. [CrossRef]

42. Whitaker, T.W.; Davis, G.N. Cucurbits. Botany, Cultivation, and Utilization; Interscience Publishers, Inc.: New York, NY, USA, 1962; p. 250.

43. Filipowicz, N.; Schaefer, H.; Renner, S.S. Revisiting Luffa (Cucurbitaceae) 25 years after C. Heiser: Species boundaries and application of names tested with plastid and nuclear DNA sequences. Syst. Bot. 2014, 39, 205-215. [CrossRef]

44. Premchand, V.; Shankarappa, K.S.; Mamatha, A.; Mesta, R.K. Screening of Luffa acutangula (L.) Roxb. genotypes for resistance against Tomato leaf curl New Delhi virus, the causal agent of yellow mosaic disease of ridge gourd. Environ. Ecol. 2017, 35, 2280-2284.

45. Criswell, A.D.; Call, A.D.; Wehner, T. Genetic Control of Downy Mildew Resistance in Cucumber-A Review. Available online: https://pdfs.semanticscholar.org/93d7/dae64056608d65835cd807eca0b6c4c6d949. pdf (accessed on 10 March 2020).

46. Epinat, C.; Pitrat, M. Inheritance of resistance to downy mildew (Pseudoperonospora cubensis) in muskmelon (Cucumis melo). I. Analaysis of a $8 \times 8$ diallel table. Agronomie 1994, 14, 239-248. [CrossRef]

(C) 2020 by the authors. Licensee MDPI, Basel, Switzerland. This article is an open access article distributed under the terms and conditions of the Creative Commons Attribution (CC BY) license (http://creativecommons.org/licenses/by/4.0/). 